\title{
Estudio para la estabilización del talud del tramo km-318+000 hasta km-318+300 de la carretera Cajamarca - Chachapoyas.
}

\author{
Study for the stabilization of the slope of the section $\mathrm{km}-318+000$ to $\mathrm{km}-318+300$ \\ of the Cajamarca - Chachapoyas highway.
}

\author{
Carlos Fernando Tafur Tuesta ${ }^{1}$
}

\section{RESUMEN}

La presente investigación tuvo por objetivo determinar una alternativa de solución para la estabilización del talud en el tramo Km 318+000 hasta Km 318+300 de la carretera Cajamarca - Chachapoyas, con el fin de lograr un tránsito seguro y permanente para el bienestar de la población. La investigación, fue descriptiva con enfoque cuantitativo, la técnica de muestreo fue no probabilística, siendo la muestra el punto más perjudicado por el deslizamiento del talud del tramo en estudio. Los resultados obtenidos del estudio topográfico fueron: la vía existente, las curvas de nivel, el área crítica y el borde del talud; del estudio de suelos fueron: para la calicata $\mathrm{N}^{\circ} 01$ un suelo gravo arcilloso, de mediana plasticidad y un contenido de humedad promedio bajo y para la calicata $\mathrm{N}^{\circ} 02$ un suelo areno-limoso, no plástico y con un contenido de humedad promedio bajo; y del análisis de estabilidad en software especializado de estabilización, para el talud inicial se tuvo un factor de seguridad máximo de 0.807 y mínimo de 0.757 , y para el talud final se tuvo un factor de seguridad máximo de 1.275 y mínimo de 1.131. Se concluye, que la alternativa de solución corresponde al movimiento masivo de tierras, a través de cortes del talud según el tipo de suelo, ya que los factores de seguridad obtenidos mayores o igual uno están en la evaluación al talud final; además, se considera una protección superficial contra erosiones, en el suelo "GC" con geomantos tipo TMR 15 y en el suelo "SP-SM" con fibra de coco.

Palabras clave: estabilización, talud, suelo, protección superficial, geomanto, fibra de coco.

\begin{abstract}
The objective of the present investigation was to determine a solution alternative for the stabilization of the slope in the section $\mathrm{Km} 318+000$ up to $\mathrm{Km} 318+300$ of the Cajamarca - Chachapoyas highway, in order to achieve a safe and permanent transit for the welfare of the population. The investigation was descriptive with a quantitative approach, the sampling technique was non-probabilistic, with the sample being the most affected by the slip of the slope of the section under study. The results obtained from the topographic study were: the existing road, the contour lines, the critical area and the edge of the slope; of the study of soils were: for pit No. 01 a gravelly clay soil, of medium plasticity and a low average moisture content and for pit No. 02 a sandy-silty, non-plastic soil with a low average moisture content; and stability analysis in specialized stabilization software, for the initial slope there was a maximum safety factor of 0.807 and a minimum of 0.757 , and for the final slope there was a maximum safety factor of 1.275 and a minimum of 1.131. It is concluded that the solution alternative corresponds to the massive earth movement, through slope cuts according to the type of soil, since the safety factors obtained greater or equal one are in the evaluation to the final slope; In addition, it is considered a superficial protection against erosions, in the soil "GC" with geomants type TMR 15 and in the soil "SP-SM" with coconut fiber.
\end{abstract}

Key words:stabilization, slope, soil, surface protection, geomanto, coconut fibre.

\footnotetext{
${ }^{1}$ Bachiller en Ingeniería Civil, correo electrónico: carlos_2407_1994@hotmail.com
} 


\section{INTRODUCCIÓN}

Se comprende bajo el nombre genérico de talud cualesquier superficie inclinada respecto a la horizontal que haya de adoptar permanentemente las estructuras de tierra, bien sea en forma natural o como consecuencia de la intervención humana en una obra de ingeniería (Pacheco, 2006).

Los proyectos de ingeniería civil (fundamentalmente obras lineales) requieren el diseño de taludes tanto en corte de terreno como en terraplén, bajo las condiciones de seguridad adecuadas. En el estudio de la estabilidad de los taludes se abordan fenómenos de estado último o de rotura de masas de suelo. El agente externo responsable de la inestabilidad es una fuerza de masa, el peso y, eventualmente, los efectos de filtración, a los cuales hay que añadir otros factores como las sobrecargas (estáticas/dinámicas) (Valiente, Sobrecases, \& Díaz, 2015).

Los primeros signos de inestabilidad de laderas son la aparición de grietas de tracción y hundimientos en la parte superior del talud y en los flancos del movimiento de ladera. Estas grietas pueden llenarse de agua, facilitando la saturación de los suelos con la consiguiente reducción de su resistencia al esfuerzo cortante; al mismo tiempo se incrementan los empujes hidrostáticos que desestabilizan la ladera (Gallardo, Guerrero, \& Macgregor, 2013).

El tramo Km 318+000 hasta Km 318+300 de la carretera Cajamarca-Chachapoyas es una vía de dos carriles que está al servicio de la población y en esta longitud de 300 metros lineales se detectó un área crítica, con una inestabilidad permanente provocando un tránsito inseguro.

En tal contexto, se formuló el problema en la interrogante siguiente: ¿Cómo evitar el deslizamiento del talud debido a la inestabilidad del suelo en el tramo $\mathrm{Km} 318+000$ hasta $\mathrm{Km}$ $318+300$ de la carretera Cajamarca Chachapoyas?.

Así mismo, la investigación se justifica en la importancia que tiene el tramo en estudio; siendo este el ingreso principal a la ciudad de Chachapoyas y que durante las épocas de lluvias intensas la inestabilidad en el talud es permanente; con esta investigación se buscó mejorar el bienestar en la población.

\section{MATERIAL Y MÉTODOS}

Objeto de estudio

Estabilización del talud.

\section{Diseño de investigación}

El presente trabajo de investigación fue de tipo descriptivo; ya que se describió los indicadores de las dimensiones de la variable de estudio.

$$
\begin{aligned}
M & \rightarrow O_{x} \\
M & =\text { Muestra. } \\
O_{x} & =\text { Observación del deslizamiento. }
\end{aligned}
$$

\section{Población}

Fue definida por el tramo $\mathrm{Km} 318+000-\mathrm{Km}$ $318+300$ de la carretera Cajamarca Chachapoyas, ya que fue el área de interés para este trabajo.

\section{Muestra}

Fue el punto más perjudicado por el deslizamiento del talud en estudio, la progresiva Km 318+103.

\section{Muestreo}

No probabilístico, muestreo por juicio del investigador. 
Métodos

> Ubicación de la zona de estudioObtenido a criterio del investigador.

Posición geográfica: El tramo en estudio de la carretera Cajamarca - Chachapoyas fue delimitada por las progresivas $\mathrm{Km} 318+000$ (coordenadas: Norte: 9310000.8, Este: 178675.6 y Altitud: 2043.0) hasta Km 318+300 (coordenadas: Norte: 9309744.8, Este: 178448.1 y Altitud: 2056.0).

Extensión territrial: 300 metros lineales.

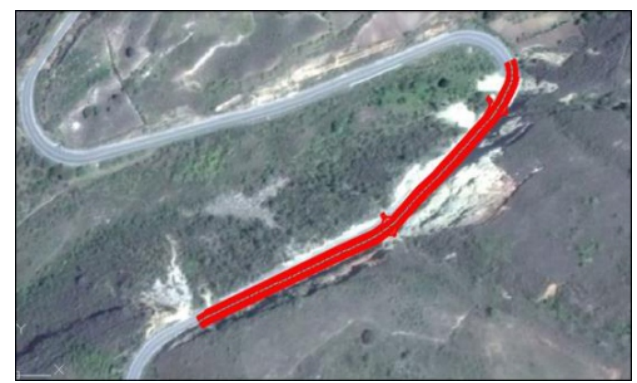

Figura $\mathrm{N}^{\circ} 01$. Ubicación del tramo en estudio

$>$ Características de la zona de estudio:E1 tramo Km 318+000 hasta $\mathrm{Km} \mathrm{318+300} \mathrm{de} \mathrm{la}$ carretera Cajamarca - Chachapoyas, se caracteriza por presentar en su talud de corte poca vegetación (bosques bajos y medianos, característicos de la zona) y pendientes pronunciadas.

Exploración realizada en campo. Los estudios realizados se localizaron en la progresiva $\mathrm{Km} 318+103$ del tramo de la carretera Cajamarca - Chachapoyas. Se optó por esta progresiva porque se consideró que es el punto donde se produce un permanente deslizamiento, ya que el aporte de este proyecto es generar un tránsito seguro en cualquier estación del tiempo para el bienestar de la población.

Definir las inclinación en la sección crítica: La inclinación del talud en relación $\mathrm{H}: \mathrm{V}$ (Horizontal:Vertical) se definió en gabinete con cortes imaginarios de acuerdo a las propiedades físico-mecánicas del suelo, bajo condiciones estáticas, a partir de un modelo de estado de equilibrio límite de la masa de suelo que conforma el área crítica, donde el indicador numérico será el factor de seguridad.

\section{Técnicas}

Esta investigación se realizó a través de

La observación directa.

\section{$\underline{\text { Instrumentos }}$}

Ficha Técnica

Fichas Bibliográficas

Procedimiento

Elección del tema.

$>$ Ubicación y delimitación del área de investigación.

$>$ Redacción del proyecto de tesis que sostiene la base de la investigación.

Con la aprobación de este tema de investigación, se empezó a redactar el informe de proyecto de tesis de acuerdo al formato establecido.

$>$ Se definió la metodología a utilizar.

Para la obtención de datos de campo, el estudio se realizó en tres etapas:

Pre Campo:

- Planificación de las actividades a realizar. 
- Recopilación de información bibliográfica referente al tema.

Campo:

- Reconocimiento inicial del tramo, en el pie y en la cresta del talud, definiendo a criterio del investigador el área crítica.

- Levantamiento topográfico del área de estudio.

- Ubicación, excavación y extracción de muestras de suelo en las dos calicatas que se hizo.

Post Campo:

- Ensayos en laboratorio de las muestras de suelo.

- Organizar, interpretar y procesar toda la información de campo.

Se analizaron y procesaron los datos obtenidos en campo y de otras fuentes, para la obtención de resultados.

Se continuó con la realización de conclusiones y recomendaciones.

$>$ Se culminó con la elaboración del informe de tesis final.

\section{Análisis de datos}

Se analizó los datos obtenidos a través de un modelamiento en el software especializado de estabilización, considerando los siguientes enfoques:

Los métodos de Equilibrio Límite.

El tipo de suelo y la topografía del tramo en estudio.

Comparación de los métodos de estabilización de taludes para seleccionar el más eficiente y recomendable.
Además:

Se utilizó gráficos de barras como indicadores estadísticos y software estadístico para la prueba de hipótesis.

Se utilizó software de diseño asistido por computadora para procesamiento de datos topográficos.

\section{RESULTADOS}

\subsection{Estudio topográfico}

En esta sección, se plasman los resultados obtenidos del levantamiento topográfico: La vía existente, las curvas de nivel, el área crítica, el borde del talud, entre otros, tal como se indican en la Figura $\mathrm{N}^{\circ} 02$.

Además, teniendo como criterio la mayor pendiente en el área crítica (área inestable) se ha tomado la sección transversal crítica, tipo para nuestro modelamiento en la fase inicial del talud (Figura $\mathrm{N}^{\circ} 03$ ) y en la fase final (Figura $\mathrm{N}^{\circ} 04$ ), es decir, cuando se ha hecho los cortes respectivos de las banquetas.

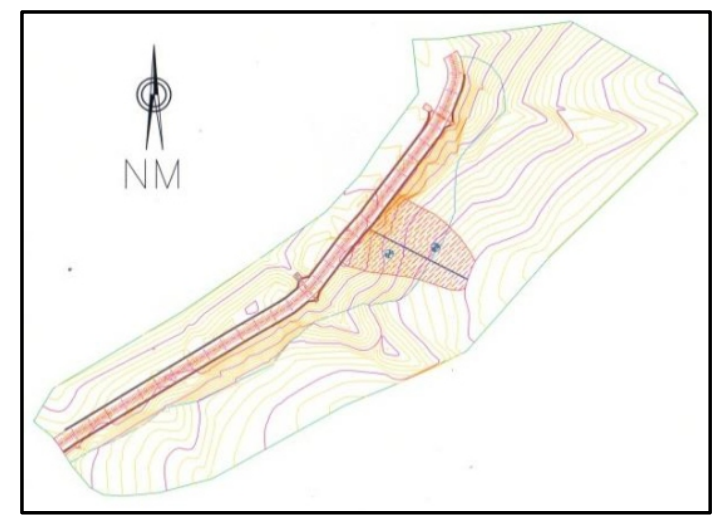

Figura $\mathrm{N}^{\circ}$ 02. Resultados del levantamiento topográfico. 


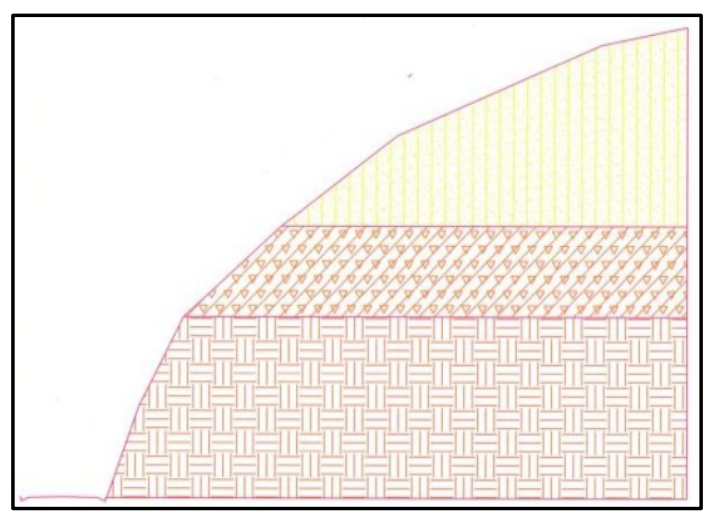

Figura $\mathrm{N}^{\circ}$ 03. Sección transversal crítica: Talud inicial.

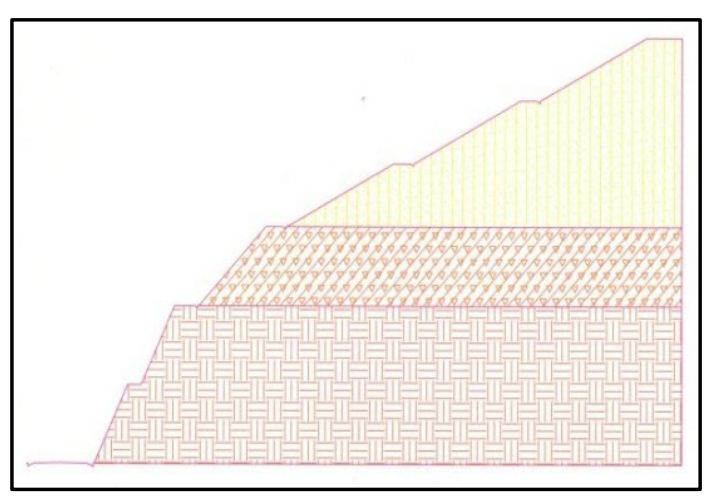

Figura $\mathrm{N}^{\circ}$ 04. Sección transversal crítica: Talud final.

\subsection{Estudio de suelos}

En la Tabla $\mathrm{N}^{\circ} 01$ se resumen las propiedades físico-mecánicas de cada una de las muestras de suelo obtenidas para la investigación y cuyas condiciones de estabilidad se investigan.

Estos resultados obtenidos en el Laboratorio de Mecánica de Suelos y Concreto de la Dirección Regional de Transportes y Comunicaciones de Amazonas, son de acuerdo a cada muestra y ensayo que se realizó.
Tabla $\mathrm{N}^{\circ}$ 01. Resultado de las propiedades físicomecánicas del suelo.

\begin{tabular}{|c|c|c|c|c|c|c|c|}
\hline \multirow{2}{*}{\multicolumn{3}{|c|}{ Estudio de Mecánica de Suelos }} & \multicolumn{5}{|c|}{ Calicata/Estrato } \\
\hline & & & \begin{tabular}{|l|} 
C-1 $/$ \\
E-01 \\
\end{tabular} & \multirow{2}{*}{\begin{tabular}{|l|} 
C-1 / \\
E-02 \\
10.35 \\
\end{tabular}} & \multirow{2}{*}{ 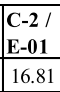 } & \multirow{2}{*}{\begin{tabular}{|c|}
$\begin{array}{c}\mathbf{C}-\mathbf{2} / \\
\mathbf{E - 0 2}\end{array}$ \\
8.83
\end{tabular}} & \multirow{2}{*}{ 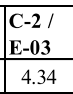 } \\
\hline Contenido de $\mathrm{Hu}$ & umedad prom & iio (\%) & 9.00 & & & & \\
\hline \multirow{5}{*}{$\begin{array}{l}\text { Análisis } \\
\text { Granulométrico } \\
\text { por tamizado }\end{array}$} & \multirow{3}{*}{ Características } & Gravas (\%) & 42.7 & 43.0 & 0.7 & 0.5 & 0.0 \\
\hline & & Arenas (\%) & 22.9 & 21.8 & 45.1 & 66.8 & 88.1 \\
\hline & & Finos (\%) & 34.4 & 35.3 & 54.2 & 32.7 & 11.9 \\
\hline & \multirow[b]{2}{*}{ Clasificación } & SUCS & $\mathrm{GC}$ & $\mathrm{GC}$ & ML & SM & SP-SM \\
\hline & & AASHTO & $\begin{array}{c}\text { A-2-6 } \\
(0)\end{array}$ & $\begin{array}{c}\text { A-6 } \\
(0)\end{array}$ & $\begin{array}{l}\text { A-4 } \\
(4)\end{array}$ & $\begin{array}{c}\text { A-2-4 } \\
(0)\end{array}$ & $\begin{array}{c}\text { A-2-4 } \\
(0)\end{array}$ \\
\hline \multirow{3}{*}{$\begin{array}{l}\text { Límites de } \\
\text { consistencia }\end{array}$} & \multicolumn{2}{|c|}{ Limite Líquido (\%) } & 27.0 & 25.0 & NP & NP & NP \\
\hline & \multicolumn{2}{|c|}{ Límite Plástico (\%) } & 16.0 & 14.0 & NP & $\mathrm{NP}$ & $\mathrm{NP}$ \\
\hline & \multicolumn{2}{|c|}{ Índice de Plasticidad (\%) } & 11.0 & 11.0 & $\mathrm{NP}$ & $\mathrm{NP}$ & NP \\
\hline Peso específico & \multicolumn{2}{|c|}{$\begin{array}{l}\text { Peso específico del suelo } \\
(\mathrm{gr} / \mathrm{cm} 3)\end{array}$} & - & 1.81 & & - & 1.62 \\
\hline \multicolumn{3}{|c|}{ Material que pasa el tamiz número $200(\%)$} & 34.42 & 35.25 & 54.15 & 32.73 & 11.87 \\
\hline \multirow{2}{*}{$\begin{array}{l}\text { Ensayo de } \\
\text { Corte Directo }\end{array}$} & \multicolumn{2}{|c|}{ Cohesión $\left(\mathrm{kg} / \mathrm{cm}^{2}\right)$} & - & 0.38 & - & - & 0.00 \\
\hline & \multicolumn{2}{|c|}{ Ángulo de fricción interna } & - & 12.10 & - & - & 32.80 \\
\hline
\end{tabular}

En esta tabla se puede describir que:

Para la Calicata $\mathrm{N}^{\circ} 01$, en sus estratos 01 y 02 , se tiene un suelo gravo arcilloso, de mediana plasticidad y con un contenido de humedad promedio bajo.

Para la Calicata $\mathrm{N}^{\circ} 02$ : En su estrato 01, se tiene un suelo de limo inorgánico con mezclas de arena gruesa, mediana y fina, y es no plástico; en su estrato 02 , se tiene un suelo de arenas limosas, no plástico y con un contenido de humedad promedio bajo y en su estrato 03 , se tiene un suelo de arena pobremente graduada con limo, con poco porcentaje de material granular, no plástico y con un contenido de humedad promedio bajo.

Las propiedades físico-mecánicas de las muestras de suelo que se ingresó al software especializado de estabilización se indican en la Figura $\mathrm{N}^{\circ} 05 \mathrm{y}$ la Figura $\mathrm{N}^{\circ} 06$. 


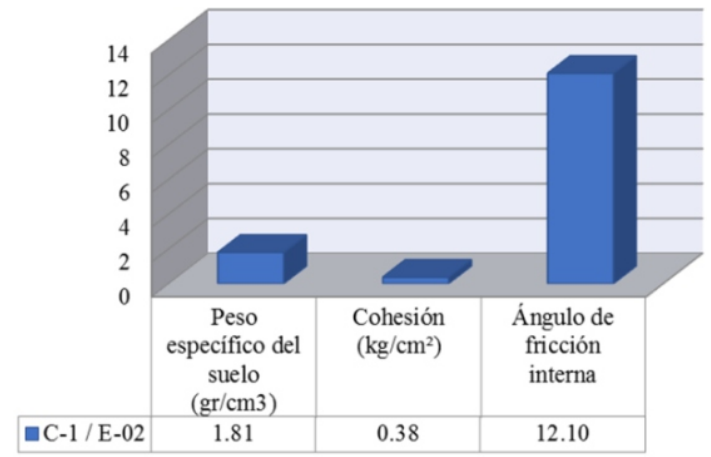

Figura $N^{\circ}$ 05. Clasificación SUCS “GC" de la muestra de suelo de la C-01/E-02.

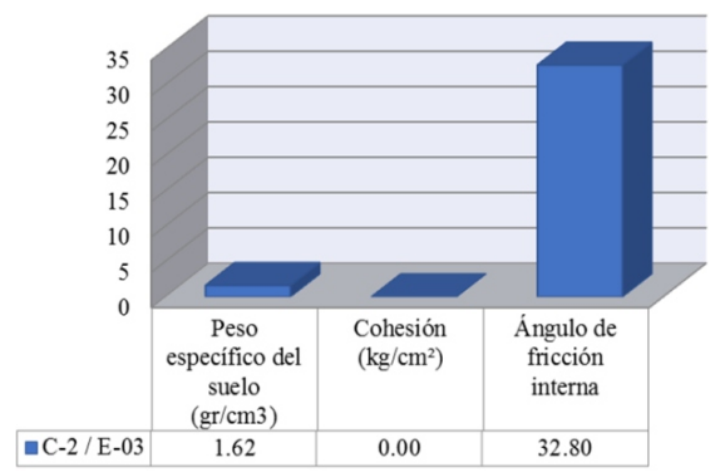

Figura $N^{\circ}$ 06. Clasificación SUCS "SP-SM" de la muestra de suelo de la C-02/E-03.

\subsection{Análisis de estabilidad en software especializado de estabilización}

\subsubsection{Resultados para el talud inicial}

En la Figura $\mathrm{N}^{\circ} 07$, se muestran los valores numericos del factor seguridad, obtenidos del análisis del talud inicial bajo condiciones estáticas en el software especializado de estabilización, a partir de un modelo de estado de equilibrio límite de la masa de suelo que conforma el área crítica.

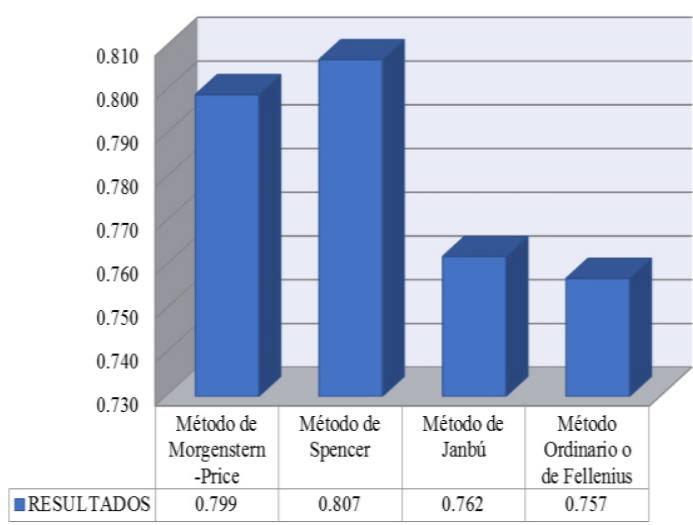

Figura $\mathrm{N}^{\circ} 07$. Resultados de factor seguridad del talud inicial.

\subsubsection{Resultados para el talud final}

Al igual que el analisis del talud inicial, se tiene las mismas consideraciones para el análisis del talud final. En la Figura $\mathrm{N}^{\circ} 08$, se muestran los valores numericos del factor seguridad, obtenidos del análisis del talud final.

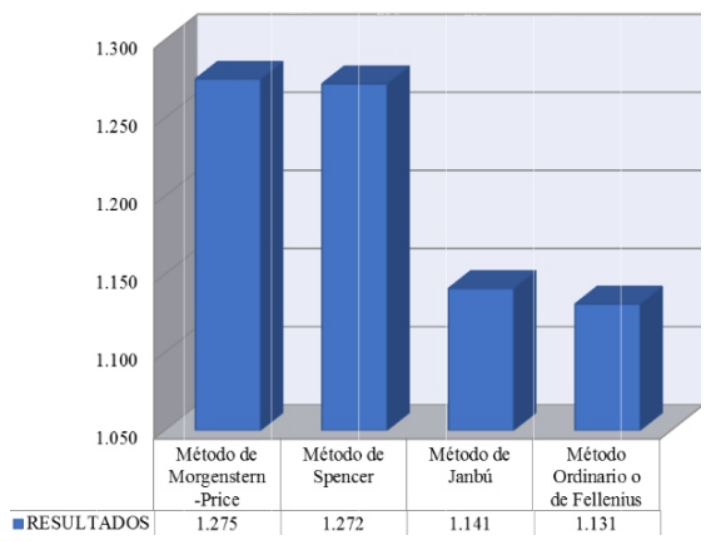

Figura $\mathrm{N}^{\circ} 08$. Resultados de factor seguridad de talud con banquetas.

\section{DISCUSIóN}

Granados (2006) afirma que para encontrar las propiedades geomecánicas de suelos gravoarcillosos y suelos arenosos, asumiendo perfiles estratigráficos horizontales homogéneos es necesario realizar ensayos de Compresión Triaxial, ya que con este ensayo se obtendrá los valores correctos de la cohesión y el ángulo de 
fricción, datos necesarios para aplicar la teoría de Mohr Coulomb y hacer un modelamiento en el software Slope. En esta investigación se hizo el ensayo de Corte Directo (similar al ensayo de Compresión Triaxial) para encontrar las propiedades geoménicas de las muestras de suelo, valores necesarios para hacer el modelamiento de la sección crítica y obtener el valor numérico del factor seguridad en el software especializado de estabilización.

Guillen (2004) afirma, que la inestabilidad se produce como resultado de la falla por esfuerzo cortante en una serie de puntos que definen una superficie, teniendo como causa principal las pendientes mayores a $50.00 \%$ en suelos arenosos o deleznables, ya que con este porcentaje de pendiente superan el ángulo de reposo del talud. En esta investigación en el análisis del talud inicial se tuvo pendientes máximas de $74.35 \%$ en suelo areno limoso, lo que nos indica que la causa principal de la inestabilidad en el talud es la pendiente actual de corte, por tal motivo se define los cortes del talud con inclinación 1:1 (Horizontal:Vertical) para la evaluación del talud final.

Salamanca (2016) afirma, que la diferencia de resultados del factor seguridad entre los métodos de Spencer y el de Morgenstern-Price es muy poca $\mathrm{y}$ que ambos métodos son muy precisos $\mathrm{y}$ prácticamente aplicable a todas las geometrías y perfiles de suelo, además son los más utilizados actualmente. En esta investigación los resultados numéricos mayores del indicador de factor seguridad son obtenidos a través de los métodos de Spencer y Morgenstern-Price, tanto en el análisis del talud inicial y el talud final, lo que nos conlleva a decir que dichos resultados son correctos.

Pacheco (2006) dice, que el método del equilibrio límite se basa en el cálculo del factor de seguridad del talud en estudio, lo cual nos indica lo siguiente: si el factor de seguridad es menor a la unidad $(\mathrm{FS}<1)$, el talud es inestable o ya colapsado y si el factor de seguridad es mayor o igual a la unidad (FS $\geq 1$ ), el talud es estable; además dice que para mejorar el factor seguridad en un talud se puede utilizar sistemas de protección o refuerzo superficial. En esta investigación se obtuvo los valores del factores de seguridad menores a uno en el análisis del talud inicial, por lo que se dice que el talud es inestable y valores del factor seguridad mayores a uno en el análisis del talud final, por lo que se dice que el talud ya es estable, además se incluyó una protección superficial contra erosiones pluviales con geomantos tipo TMR 15 para el tipo de suelo "GC" y con fibra de coco para el tipo de suelo "SP-SM".

\section{CONCLUSIONES}

La alternativa para la estabilización del talud, se planteó a partir del movimiento masivo de tierras, para suelos "GC" con banquetas cada $10.00 \mathrm{~m}$ de altura, con una inclinación del talud 1:1 (Horizontal: Vertical) y para suelos "SP-SM" con banquetas cada $8.00 \mathrm{~m}$ de altura, con una inclinación del talud del 1.5:1 (Horizontal: Vertical); además sobre la cresta de corte para cada tipo de suelo se propuso una banqueta de $3.00 \mathrm{~m}$ de ancho, donde está incluido una cuneta de coronación triangular revestida con un ancho de $0.50 \mathrm{~m}$ y una profundidad de $0.25 \mathrm{~m}$. 
Se logró el levantamiento topográfico de toda la extensión territorial del talud en estudio a través de una estación total, este equipo topográfico nos facilitó encontrar las coordenadas y cota de los puntos que conforman la superficie en base a un punto conocido.

Las propiedades físico-mecánicas del suelo del talud, en la calicata 01/estrato 02 son: clasificación SUCS $=\mathrm{GC}$, peso específico $(\mathrm{gr} / \mathrm{cm} 3)=1.81$, cohesión $\left(\mathrm{kg} / \mathrm{cm}^{2}\right)=0.38$, ángulo de fricción interna $=12.10 ; \mathrm{y}$, en la calicata 02/estrato 03 son: clasificación SUCS $=$ SP-SM, peso específico $(\mathrm{gr} / \mathrm{cm} 3)=1.62$, cohesión $\left(\mathrm{kg} / \mathrm{cm}^{2}\right)=0.00$, ángulo de fricción interna $=$ 32.80 .

El método analítico de equilibrio límite que se determinó para la estabilización del talud es el de Morgenstern-Price, con un indicador del factor seguridad de 1.275.

La protección superficial contra erosiones pluviales para el suelo tipo "GC" fue con geomantos tipo TMR 15 y, para el suelo tipo "SPSM" con fibra de coco. Esto se definió a partir de los resultados de suelos obtenidos y la recomendación de Cidelsa (Empresa especializada en productos para reforzamiento y protección de taludes).

\section{REFERENCIA BIBLIOGRÁFICA}

Gallardo, R., Guerrero, T., \& Macgregor, A. (2013). Investigación geotécnica para la estabilización de las laderas del barrio San Fermín, municipio de Ocaña, departamento de Norte de Santander (Colombia). INGE CUC, 66-74.
Granados, A. (2006). Estabilización del talud de la Costa Verdeen la zona del distrito de Barranco. (Tesis de Ingeniero). Pontificia Universidad Católica. Lima, Perú.

Pacheco, A. (2006). Estabilización del talud de la Costa Verde en la zona del distrito de San Isidro. (Tesis de Ingeniero). Pontificia Universidad Católica. Lima, Perú.

Salamanca, I. (2016). Estudio de la estabilidad de muros ecológicos con geotextiles mediante métodos tenso deformacionales. Técnica de diseño y predicción de fallos. (Tesis de Ingeniero). Universidad de Sevilla. Sevilla, España.

Valiente, R., Sobrecases, S., \& Díaz, A. (2015). Estabilidad de taludes: conceptos básicos, parámetros de diseño y métodos de cálculo. CIVILIZATE $\mathbf{N}^{\mathbf{0}} .7$ 